\title{
Severe corneal melting and perforation with dacryocystitis due to decreased ophthalmology consultation during COVID-19 pandemic
}

\author{
Daisuke Nagasato $^{1}$, Hitoshi Tabuchi ${ }^{1}$, Tomofusa Yamauchi ${ }^{1}$, Hitoshi Imamura ${ }^{1}$, and Yoshie \\ Shimizu ${ }^{1}$ \\ ${ }^{1}$ Tsukazaki Hospital
}

July 7,2020

\begin{abstract}
A patient with dacryocystitis who had a regular ophthalmology visit canceled the regular visit with a COVID-19 pandemic. After that, serious deterioration was observed and emergency evisceration was performed. Even during COVID-19 pandemic, so it is necessary to continue regular eye examinations and infection treatment.
\end{abstract}

\section{CLNICAL IMAGE}

A 79-year-old man receiving regular, once-monthly ophthalmologic treatment for left dacryocystitis had normal cornea on March 10, 2020. On April 5, he noticed decreasing left visual acuity; however, fear of contracting coronavirus disease 2019 (COVID-19) led him to cancel his scheduled appointment on April 7. On May 11, he noticed significant left visual impairment with severe pain and promptly visited the ophthalmology clinic. As left corneal perforation was observed, he was urgently referred to our hospital. His left cornea has melted and was perforated from the center to the lower part, exposing the intraocular lens and iris (Figure). Methicillin-sensitive Staphylococcus aureus (MSSA) was detected in a culture test. Emergency evisceration was performed. Fluoroquinolone antibacterial eye drops, commonly used as antibacterial agents, have strong antibacterial activity against pathogenic bacteria causing various bacterial infections including MSSA. ${ }^{1,2}$ If he had attended the scheduled appointment on April 7 and had received routine ophthalmologic examination and infection treatment, his visual function would probably have been preserved.

This case underscores the necessity of maintaining ophthalmologic consultations during the COVID-19 pandemic.

\section{CONFLICT OF INTEREST}

None declared.

\section{ACKNOWLEDGMENTS}

We thank Masayuki Miki at Tsukazaki Hospital for support the image creation.

\section{AUTHOR CONTRIBUTIONS}

Daisuke Nagasato, MD, PhD has managed the patient, wrote the manuscript and critically reviewed the manuscript, references and images. Hitoshi Tabuchi, $\mathrm{MD}, \mathrm{PhD}$ has reviewed the manuscript. Tomofusa Yamauchi, MD, PhD has reviewed the manuscript. Hitoshi Imamura, MD, PhD managed the patient. Yoshie Shimizu, MD, PhD managed the patient.

\section{REFERENCES}


1. Hosaka, M., Yasue, T., Fukuda, H., Tomizawa, H., Aoyama, H., Hirai, K. In vitro and in vivo antibacterial activities of AM-1155, a new 6-fluoro-8-methoxy quinolone. Antimicrobial Agents and Chemotherapy 1992;36 :2108-2117.

2. Kowalski, R. P., Dhaliwal, D. K., Karenchak, L. M., et al. Gatifloxacin and moxifloxacin: an in vitro susceptibility comparison to levofloxacin, ciprofloxacin, and ofloxacin using bacterial keratitis isolates. American Journal of Ophthalmology2003;136:500-505.

\section{Figure}

Slit-lamp photograph of the patient's left eye at the time of his hospital visit

The lower part of the cornea is melted. The central part of the cornea is perforated with an extruded intraocular lens and a prolapsed iris (indicated by the arrow).

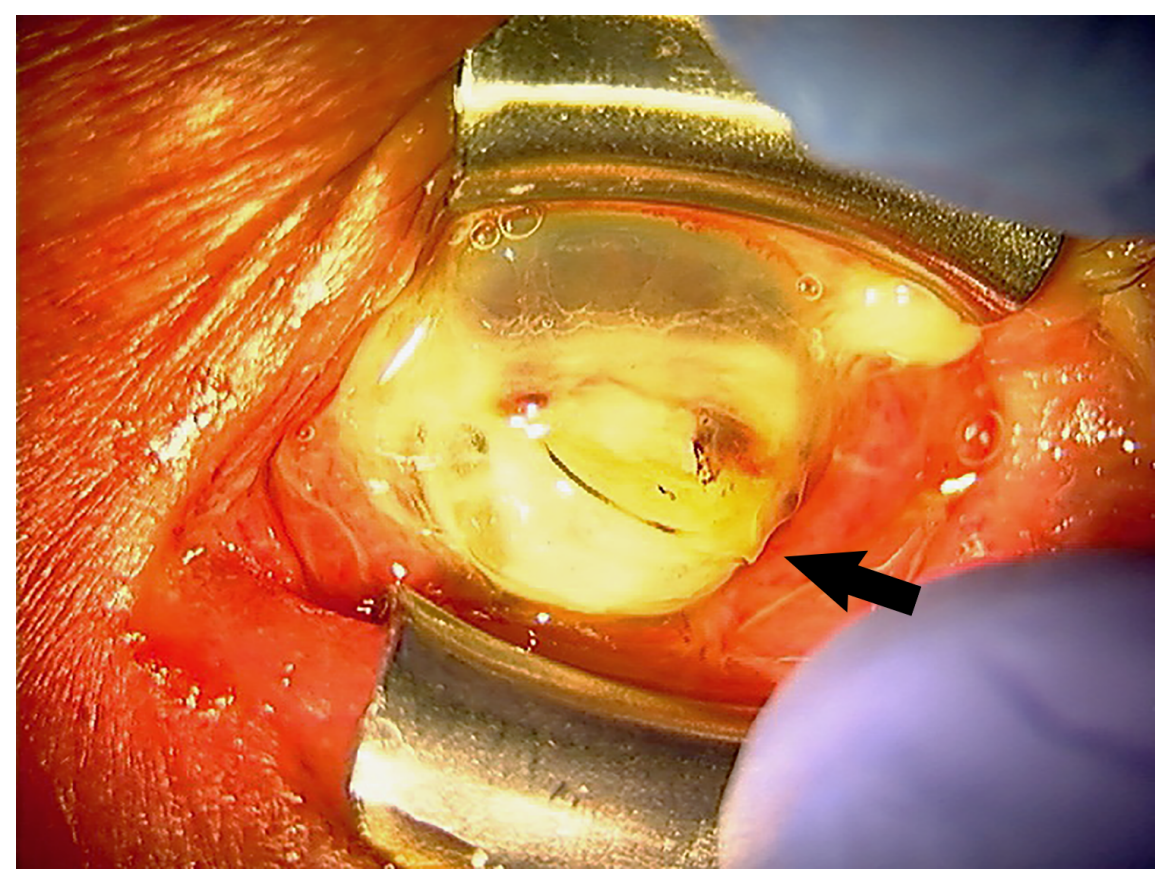

